\title{
Hybrid Quantum Repeater Using Bright Coherent Light
}

\author{
P. van Loock,, , 田 T. D. Ladd, ${ }^{2,3}$ K. Sanaka, ${ }^{2,3}$ F. Yamaguchi, ${ }^{2}$ Kae Nemoto, ${ }^{1}$ W. J. Munro, ${ }^{4,1}$ and Y. Yamamoto ${ }^{1,2}$ \\ ${ }^{1}$ National Institute of Informatics, 2-1-2 Hitotsubashi, Chiyoda-ku, Tokyo 101-8430, Japan \\ ${ }^{2}$ Edward L. Ginzton Laboratory, Stanford University, Stanford, California 94305-4088, USA \\ ${ }^{3}$ Nanoelectronics Collaborative Research Center, IIS, University of Tokyo, Tokyo 153-8505, Japan \\ ${ }^{4}$ Hewlett-Packard Laboratories, Filton Road, Stoke Gifford, Bristol BS34 8QZ, United Kingdom
}

\begin{abstract}
We describe a quantum repeater protocol for long-distance quantum communication. In this scheme, entanglement is created between qubits at intermediate stations of the channel by using a weak dispersive light-matter interaction and distributing the outgoing bright coherent light pulses among the stations. Noisy entangled pairs of electronic spin are then prepared with high success probability via homodyne detection and postselection. The local gates for entanglement purification and swapping are deterministic and measurement-free, based upon the same coherent-light resources and weak interactions as for the initial entanglement distribution. Finally, the entanglement is stored in a nuclear-spin-based quantum memory. With our system, qubit-communication rates approaching $100 \mathrm{~Hz}$ over $1280 \mathrm{~km}$ with fidelities near $99 \%$ are possible for reasonable local gate errors.
\end{abstract}

PACS numbers: 03.67.Hk, 03.67.Mn, 42.50.Pq

In a quantum repeater, long-distance entanglement is created by distributing entangled states over sufficiently short segments of a channel such that the noisy entangled states in each segment can be purified and connected via entanglement swapping [1, 2]. The resulting entanglement between the qubits at distant stations can then be used, for example, to teleport quantum information [3] or transmit secret classical information [4]. Existing approaches to quantum repeaters generate entanglement using postselection with single-photon detection [5, 6, 7]. In these schemes, high-fidelity entanglement is created and the subsequent entanglement purification is needed primarily to compensate the degrading effect of connecting the imperfect entangled pairs via swapping. However, due to their rather low success probabilities in the initial entanglement distribution, these protocols feature very low communication rates.

More efficient schemes, compatible with existing classical optical communication networks, would involve bright multi-photon signals. In this Letter, we propose such a scheme that operates in a regime of modest initial fidelities, but creates entangled states at high speed. The high rate in the generation of entangled pairs is mainly due to the near-unit efficiencies for the homodyne detection of bright signals, as opposed to the low efficiencies of single-photon detectors. In our scheme, the resulting entangled pairs will be discrete atomic qubit states, but the probe system we use is a bright light pulse described and measured via a continuous phase observable; hence, our quantum repeater is "hybrid" not only because it employs matter signals and light probes (as in other schemes), but more distinctly, by utilizing both discrete and continuous quantum variables.

In general, in order to realize universal quantum computation or, more relevant to us here, long-distance quantum communication, a nonlinear element is needed for the implementation. Optically, this nonlinear element may be introduced in at least two possible ways. The first method uses only linear transformations, but a measurement-induced nonlinearity 8]. In the second approach, linear gates are supplemented by a weak nonlinear gate where the nonlinearity is effectively enhanced through a sufficiently strong probe beam [9]. Here we will apply this concept to quantum communication by considering a hybrid system based on optical carrier waves, electron-spin signals, and nuclear-spin memories. In our proposal, a bright coherent "probe" pulse sequentially interacts with two electronic spins placed in cavities at neighboring repeater stations. Entangled qubit pairs will then be postselected conditioned upon the results of probe homodyne measurements. Despite this postselection, high success probabilities can still be achieved, thus keeping the main advantage of our proposal over the single-photon-detection based protocols [6, 7]. We will also avoid the complication of purifying an atomic ensemble [5] and directly distill the entanglement from several copies of noisy entangled electronic-spin pairs.

The electronic and nuclear spin systems may be achieved, for example, by single electrons trapped in quantum dots 10] or by neutral donor impurities in semiconductors [11]. For a sufficient interaction between the electron and the light, the system should be placed in a cavity resonant with the light; for the cavity, weak coupling is sufficient, but a high value of $Q / V$ is required, where $Q$ is the quality and $V$ is the mode-volume of the cavity [12]. The entire quantum repeater scheme proposed here, including entanglement distribution, purification, and swapping, will be based on the same bright coherent-light resources and weak interactions.

The mechanism for the entanglement distribution among the nearest stations of the channel is illustrated in Fig. 1] The electron spin system in the cavity is treated as a $\Lambda$-system, with two stable or metastable ground states $|0\rangle$ and $|1\rangle$, only one of which $(|1\rangle)$ participates in the 


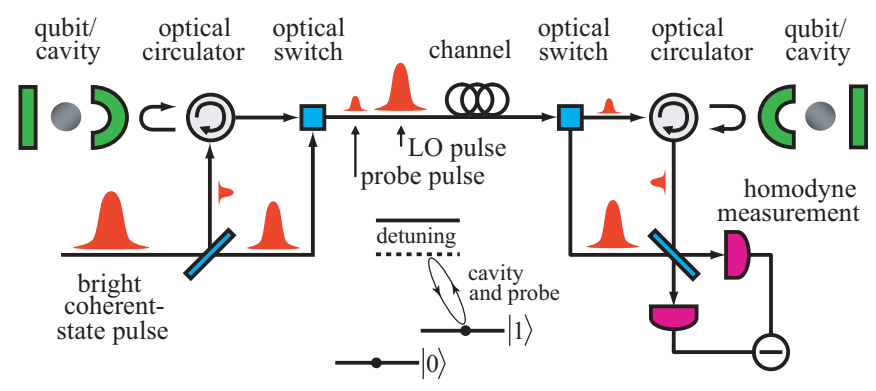

FIG. 1: Schematic for the generation of spin-entanglement between two qubits at neighboring stations via homodyne detection discriminating between conditionally phase-rotated coherent probe beams; the LO pulse is a sufficiently strong local oscillator used for the homodyne detection.

interaction with the cavity mode. Local rotations between states $|0\rangle$ and $|1\rangle$ may be achieved via stimulated Raman transitions; in particular, we suppose the state is initially prepared in the state $(|0\rangle+|1\rangle) / \sqrt{2}$. The probe light is sufficiently detuned from the transition between $|1\rangle$ and the excited state to allow for a strictly dispersive light-matter interaction. The finite probability for spontaneous emission of the qubit and for light to leak from the cavity add a small correction to channel losses, which we consider shortly. For clarity, let us first discuss entanglement distribution in the absence of loss. When the probe beam in coherent state $|\alpha\rangle$ reflects from the cavity, the total output state may be described by

$$
\hat{U}_{\text {int }}[(|0\rangle+|1\rangle)|\alpha\rangle] / \sqrt{2}=\left(|0\rangle|\alpha\rangle+|1\rangle\left|\alpha e^{-i \theta}\right\rangle\right) / \sqrt{2} \text {. }
$$

For semiconductor impurities and realistic cavity parameters, phase shifts of $\theta \sim 0.01$ are achievable 12]. After acquiring such a conditional phase shift at one station, the probe beam is sent to a neighboring station and interacts with a second spin in a similar way. Applying a further linear phase shift of $\theta$ to the probe will yield the total state $\left(\sqrt{2}\left|\Psi^{+}\right\rangle|\alpha\rangle+|00\rangle\left|\alpha e^{i \theta}\right\rangle+|11\rangle\left|\alpha e^{-i \theta}\right\rangle\right) / 2$, where $\left|\Psi^{+}\right\rangle=(|01\rangle+|10\rangle) / \sqrt{2}$. Thus, by discriminating a zero-phase shift from a $\pm \theta$ phase shift for the probe, one can project the two spins onto a maximally entangled state [9, 13]. Assuming $\alpha$ real, such a projection can be approached via a $p$ quadrature measurement (i.e., along the imaginary axis in phase space), postselecting the desired $\left|\Psi^{+}\right\rangle$state.

With the "momentum" quadrature operator defined as $\hat{p}=\left(\hat{a}-\hat{a}^{\dagger}\right) / 2 i$, the conditional state of the spin system for a measured $p$ value of the probe beam may now be written as

$$
\left|\psi^{\mathrm{C}}(p)\right\rangle=\frac{C_{0}(p)}{\sqrt{2}}\left|\Psi^{+}\right\rangle+\frac{C_{1}(p)}{2}|00\rangle+\frac{C_{-1}(p)}{2}|11\rangle,
$$

where $C_{s}(p) \equiv G_{s}(p) K_{s}(p), s=0, \pm 1$, is a Gaussian amplitude function with $G_{s}(p)=(2 / \pi)^{1 / 4} \exp \left[-(p-s d)^{2}\right]$ and the phase factors $K_{0}(p)=\exp (-2 i \alpha p), K_{r}(p)=$ $\exp [-i \alpha \cos \theta(2 p-r d)], r= \pm 1$. In order to assess our ability to distinguish the desired $\left|\Psi^{+}\right\rangle$state (around zero phase shift of the probe) and the two unwanted terms corresponding to the two phase-rotated probe beams, we consider the distance of the corresponding Gaussian peaks along the $p$ axis, $d \equiv \alpha \sin \theta$. In the following, this parameter $d$ is referred to as the distinguishability. The maximally entangled state is postselected by keeping the state only when the measured result $p$ is within some finite measurement window, $|p|<p_{c}$. Were it not for optical losses, a very large window could be chosen, because by increasing $\alpha$, the distinguishability could be made even larger, resulting in nearly perfect postselection with probability of success $1 / 2$. However, in the presence of loss, there will be a trade-off between distinguishability and decoherence, which we now discuss.

In the presence of channel loss (and a small contribution from cavity losses and spontaneous emission), the distinguishability cannot be made arbitrarily large without suffering from decoherence. We may model the photon loss by considering a beam splitter in the channel that transmits only a part of the probe beam with transmis$\operatorname{sion} \eta^{2}$. The lost photons provide "which-path" information, and tracing over them introduces the decoherence. After the homodyne detection of the probe, the spins are described by an unnormalized conditional density matrix $\hat{\rho}^{\mathrm{C}}(p)$ which depends on the measurement result $p$ and has the following diagonal elements:

$$
\begin{aligned}
\left\langle\Psi^{ \pm}\left|\hat{\rho}^{\mathrm{C}}(p)\right| \Psi^{ \pm}\right\rangle= & \left|C_{0}(p)\right|^{2} \operatorname{Re}\left(1 \pm e^{-\gamma+i \xi}\right) / 4 \\
\left\langle\Phi^{ \pm}\left|\hat{\rho}^{\mathrm{C}}(p)\right| \Phi^{ \pm}\right\rangle= & \left(\left|C_{1}(p)\right|^{2}+\left|C_{-1}(p)\right|^{2}\right) / 8 \\
& \pm e^{-\gamma} \operatorname{Re}\left[e^{i \xi} C_{1}(p) C_{-1}^{*}(p)\right] / 4
\end{aligned}
$$

for the Bell states $\left|\Psi^{ \pm}\right\rangle=(|01\rangle \pm|10\rangle) / \sqrt{2}$ and $\left|\Phi^{ \pm}\right\rangle=$ $(|00\rangle \pm|11\rangle) / \sqrt{2}$. In the functions $C_{s}(p), \alpha$ should now be replaced by $\eta \alpha$ and $d$ should become $\eta d$. The decoherence in the channel leads to a damping factor determined by $\gamma=\alpha^{2}\left(1-\eta^{2}\right)(1-\cos \theta) \approx \frac{1}{2}\left(1-\eta^{2}\right) d^{2}$ and an extra phase $\xi \equiv \alpha^{2}\left(1-\eta^{2}\right) \sin \theta$.

In order to maximize the distinguishability of the probe states, we cannot simply make $d$ arbitrarily large. A correspondingly large $d$ value would be accompanied by an increase of the decoherence effect. This is reflected by the $d$-dependence of the loss parameter $\gamma$. The parameter $\xi$ determines a phase rotation, independent of the measurement result, which can be locally removed via static phase shifters. Thus, we set $\xi \equiv 0$.

Since we cannot make $d$ arbitrarily large, we are forced to choose a sufficiently small window for the postselection, thus making $p_{c}$ sufficiently small. This will lead to a decreasing success probability. The success probability can be calculated as

$P_{\mathrm{s}}=\operatorname{Tr} \int_{-p_{c}}^{+p_{c}} d p \hat{\rho}^{\mathrm{C}}(p)=\frac{\operatorname{erf}\left(b_{0}\right)}{2}+\frac{\operatorname{erf}\left(b_{1}\right)}{4}+\frac{\operatorname{erf}\left(b_{-1}\right)}{4}$,

using the diagonal elements of $\hat{\rho}^{\mathrm{C}}(p)$ from Eq. (3) and 


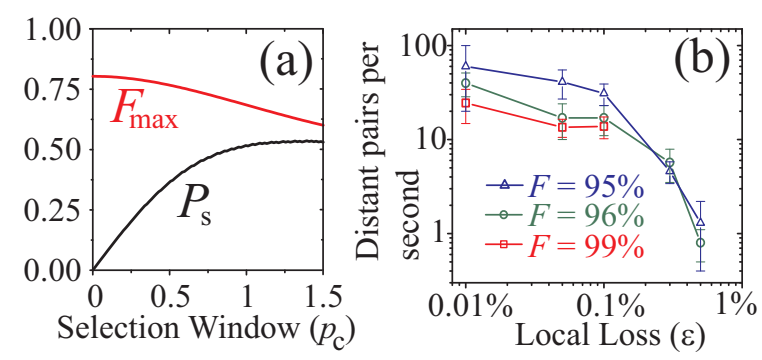

FIG. 2: (a) the maximum fidelity $F_{\max }$ and success probability $P_{\mathrm{s}}$ as a function of the postselection window $p_{c}$ for $\eta^{2}=2 / 3$. (b) achievable qubit rates for different target fidelities vs. local optical losses $\epsilon$. Each point corresponds to a single Monte-Carlo simulation of the nested purification protocol over 9 complete qubit teleportations; each point is the average difference in time between teleported qubit arrival times, and the error bar is the standard deviation.

$b_{s} \equiv \sqrt{2}\left(p_{c}+s \eta d\right), s=0, \pm 1$. The desired entangled output state is $\left|\Psi^{+}\right\rangle$, so the average fidelity after postselection becomes

$$
\begin{aligned}
F & =\frac{1}{P_{\mathrm{s}}}\left[\int_{-p_{c}}^{+p_{c}} d p\left\langle\Psi^{+}\left|\hat{\rho}^{\mathrm{C}}(p)\right| \Psi^{+}\right\rangle\right] \\
& =\frac{\operatorname{erf}\left(b_{0}\right)\left(1+e^{-\gamma}\right)}{2 \operatorname{erf}\left(b_{0}\right)+\operatorname{erf}\left(b_{1}\right)+\operatorname{erf}\left(b_{-1}\right)} .
\end{aligned}
$$

Channel loss may come from a variety of sources, including finite mode-coupling efficiency, but is likely to be dominated by fiber loss. Here, we will consider only the fiber loss 14]. A reasonable length for the individual segments of the quantum repeater would be $\ell=10 \mathrm{~km}$. Assuming telecom fiber and wavelength, where losses are about $0.17 \mathrm{~dB} / \mathrm{km}$, the transmission parameter for $10 \mathrm{~km}$ is $\eta^{2}=2 / 3$. In Fig. 2(a), the maximum fidelity is shown as a function of $p_{c}$ for a transmission of $\eta^{2}=2 / 3$. Due to the trade-off between distinguishability and decoherence, there is an optimal $d$-value yielding this maximum fidelity for each $p_{c}$. The overall maximum fidelity of $F \approx 0.8$ for $p_{c} \rightarrow 0$ can be achieved only at the expense of a vanishing success probability. However, by choosing a postselection window $p_{c} \approx 0.5$ and sacrificing some fidelity, $F \approx 0.77$, we can attain a reasonable success probability of $P_{\mathrm{s}} \approx 36 \%$. This high rate of successful entanglement generation in our scheme is in sharp contrast to the low efficiencies of single-photon-based approaches 6 , 7]. The above values for fidelity and success probability correspond to distinguishabilities of $d \sim 1$, which for phase shifts of $\theta \sim 10^{-2}$ are achievable via reasonable probe photon numbers of about $10^{4}$.

Our initial fidelities, $F_{\text {initial }} \approx 0.77$, will be insufficient for entanglement swapping; some entanglement purification must first occur. For both purification and swapping, local two-qubit gates are needed. For this purpose, we propose to use a measurement-free deterministic controlled-phase gate based upon a sequence of con- ditional rotations and unconditional displacements of a coherent-state probe interacting with the two spins 15 . The total unitary operator to achieve this gate can be described by

$$
\hat{U}_{2}(\theta) \hat{D}(\beta) \hat{U}_{1}(\theta) \hat{D}\left(-\beta^{*}\right) \hat{U}_{2}(\theta) \hat{D}(-\beta) \hat{U}_{1}(\theta) .
$$

Here, $\hat{U}_{k}(\theta)$ corresponds to the interaction in Eq. (1), leading to a controlled phase shift of the probe conditioned upon the state of the $k$ th qubit. The operator $\hat{D}(\beta)=\exp \left(\beta \hat{a}^{\dagger}-\beta^{*} \hat{a}\right)$ describes a phase-space displacement of the probe by $\beta \equiv \alpha(1-i)$. These gate operations can be implemented using the same bright coherent-light resources and weak interactions as employed in the above entanglement distribution protocol. After the entire sequence in Eq. (5), the probe will be nearly disentangled from the spins. After tracing over the probe and removing single-qubit $Z$-rotations, the qubits have undergone a controlled phase shift of $\phi \approx T(1+T) \alpha^{2} \theta^{2}$, where $T$ is the transmission for the cavity-probe interaction. For a desired phase shift of the order of $\pi$, we must satisfy $\alpha^{2} \theta^{2} \approx 1$, which is exactly the regime we have been using for the entanglement distribution. For any finite optical loss, some decoherence will occur at order $\epsilon=1-T$. A small amount of decoherence is also introduced due to the finite probe-qubit entanglement, scaling as $\theta^{2}$ if loss is neglected. The details of these decoherence mechanisms will be discussed elsewhere 12].

This controlled-sign gate, in addition to single-qubit rotations and measurements (which may also be done by homodyne detection of a bright optical probe), are sufficient resources for the standard purification protocol introduced in Ref. 16. This protocol was analyzed in terms of density matrices $\hat{\rho}$ that are exactly diagonal in the Bellstate basis. The $\hat{\rho}^{\mathrm{C}}$ described by Eq. (3) is very nearly so, and the small off-diagonal elements quickly vanish after a few purification steps. It was previously noted [2] that this protocol converges faster than protocols based on Werner states [17].

Several protocols for combining entanglement purification and swapping to connect large distances have been previously considered. At one extreme in the number of qubit resources is "scheme B" of Dür et al. [2]. This scheme uses as many qubits as are needed to allow rapid parallel purification; for example, for communication over $1000 \mathrm{~km}$, hundreds of qubits are needed in each repeater station. At the other extreme is the scheme of Childress et al. 6, 7] requiring only two qubits per station. However, in this scheme, the purification and swapping are very slow and become impossible if the initial pair-fidelities are too low and gate errors are too high.

We consider a protocol in between these two extremes; we find that for a number of qubits per station which grows only logarithmically with distance, a reasonable communication qubit-rate may be achieved for reasonable gate errors. In this scheme, each repeater station acts autonomously according to a simple set of rules. 
Throughout the protocol, all stations containing unentangled qubits simultaneously send pulses in order to immediately attempt entanglement distribution at nearestneighbor stations. Meanwhile, all entangled qubit pairs are purified a predetermined number of steps, and once this is complete, entanglement swapping occurs to progressively double the distance over which pairs are entangled. After entanglement swapping, purification is again attempted, always simultaneous with new entanglement creation at the free qubits. The limiting timescale for these operations is the time for light to propagate between stations in order to transmit both the entangling pulses and the classical signals containing measurement results for entanglement postselection, purification, and swapping.

This scheme is similar to "scheme C" of Ref. 2, where the maximum number of qubits needed per station was shown to be $2 \log _{2}(\mathcal{L} / \ell)$. Here $\mathcal{L}$ is the total length of the channel and $\ell$ is the distance between stations. However, because of the added purification needed prior to any entanglement swapping, we require at least $N=$ $2+2 \log _{2}(\mathcal{L} / \ell)$ qubits per station. We also find that the probabilistic creation of initial entangled pairs proceeds more quickly if $N$ qubits are present at every station.

As examples, we have run Monte Carlo simulations for communication over $1280 \mathrm{~km}$ with repeater stations separated by $10 \mathrm{~km}$, in which case we assume 16 qubits per station. We try several choices for the number of purification steps before and after each entanglement-swapping step. If more purification steps are used, larger fidelities are possible at slower rates, while fewer purification steps lead to faster rates at smaller final fidelities. Both the rates and fidelities drop due to local gate errors. For our simulations we presume that these errors are dominated by local optical loss. Figure 2(b) shows typical rates for different target fidelities and different amounts of local optical loss.

Two more technical issues should be raised. First, the time for optical information to propagate over $1280 \mathrm{~km}$ in optical fibers, about $6 \mathrm{~ms}$, is already longer than decoherence times observed in most solid-state electronic spin systems; to this one must add the extra time required to await the entanglement purification and swapping. A feasible solution is the introduction of nuclear memory, as decoherence times for isolated nuclei are at least many seconds [18]. For isolated nuclei, fast ENDOR (electronnuclear double resonance) pulse techniques may be employed for rapid storage and retrieval of the electron-spin state [19]. Nuclear ensembles in quantum dots have also been considered 20], but in this case the decouplinglimited memory time is likely to be shorter. The second technical consideration is that for the loss-rates over the long-distance communication channel, we have assumed telecom wavelengths, while the solid-state emitter is likely to operate at shorter wavelengths. Hence efficient phase-preserving wavelength conversion of the strong optical probe is required [21].

In summary, we proposed a full quantum repeater system based upon weak dispersive light-matter interactions. In our proposal, bright coherent light pulses interact with small numbers of solid-state electronic spin qubits in microcavities. The measured light observable is a continuous phase as opposed to a discrete occupation number. Thus, interferometric requirements are less stringent than in many other proposals and good phase stabilization is readily available from a phase-reference pulse traveling down the same fiber. For the resulting high detection efficiencies and modest initial fidelities, long-distance qubit communication rates approaching $100 \mathrm{~Hz}$ with final fidelities of $99 \%$ are possible.

The authors thank Lily Childress for useful discussions. This work was supported in part by the JSPS, MIC, Asahi-Glass research grants, the EU project QAP, JST SORST, "IT Program" MEXT, and MURI grant\# ARMY, DAAD 19-03-1-0199.

* Electronic address: vanloock@nii.ac.jp

[1] H.-J. Briegel et al., Phys. Rev. Lett. 81, 5932 (1998).

[2] W. Dür et al., Phys. Rev. A 59, 169 (1999).

[3] C. H. Bennett et al., Phys. Rev. Lett. 70, 1895 (1993).

[4] A. K. Ekert, Phys. Rev. Lett. 67, 661 (1991).

[5] L. M. Duan et al., Nature 414, 413 (2001).

[6] L. Childress et al., eprint: quant-ph/0410123 (2005).

[7] L. Childress et al., eprint: quant-ph/0502112 (2005).

[8] E. Knill, R. Laflamme, and G. J. Milburn, Nature 409, 46 (2001).

[9] K. Nemoto and W. J. Munro, Phys. Rev. Lett. 93, 250502 (2004).

[10] A. S. Bracker et al., Phys. Rev. Lett. 94, 047402 (2005).

[11] S. Strauf et al., Phys. Rev. Lett. 89, 177403 (2002); K-M. C. Fu et al., Phys. Rev. B 69, 125306 (2004).

[12] T. D. Ladd et al., in preparation.

[13] W. J. Munro, K. Nemoto, and T. P. Spiller, New J. Phys. 7, 137 (2005).

[14] Other sources of loss such as finite fiber-cavity coupling efficiencies could also be included into our loss analysis, because modeling loss via a single transmission parameter $\eta^{2}$ and tracing out lost photons will always yield lower bounds on the achievable fidelities.

[15] T. P. Spiller et al., New J. Phys. 8, 30 (2006).

[16] D. Deutsch et al., Phys. Rev. Lett. 77, 2818 (1996).

[17] C. H. Bennett et al., Phys. Rev. Lett. 76, 722 (1996).

[18] T. D. Ladd et al., Phys. Rev. B 71, 014401 (2005).

[19] F. Jelezko et al., Phys. Rev. Lett. 93, 130501 (2004).

[20] J. M. Taylor, C. M. Marcus, and M. D. Lukin, Phys. Rev. Lett. 90, 206803 (2003).

[21] C. Langrock et al., Opt. Lett. 30, 1725 (2005). 